\title{
Aberrant promoter methylation may be responsible for the control of CD146 (MCAM) gene expression during breast cancer progression*
}

\author{
Paulina Dudzik1, Sonia E. Trojan1, Barbara Ostrowska1, Małgorzata Lasota², \\ Joanna Dulińska-Litewka ${ }^{1}$, Piotr Laidler ${ }^{1}$ and Kinga A. Kocemba-Pilarczyk ${ }^{1}$ \\ 1Jagiellonian University - Medical College, Faculty of Medicine, Chair of Medical Biochemistry, Kraków, Poland; 2Jagiellonian University - Medical \\ College, Faculty of Medicine, Department of Transplantation, Kraków, Poland
}

The CD146 (also known as MCAM, MUC-18, Mel-CAM) was initially reported on in 1987, as a protein crucial for melanoma invasion. Recently, it has been confirmed that CD146 is involved in progression and poor overall survival of many other cancers, including breast cancer. Importantly, in independent studies, CD146 was reported to be a trigger of epithelial to mesenchymal transition in breast cancer cells. The goal of our current study was to verify possible involvement of an epigenetic mechanism behind regulation of the CD146 expression in breast cancer cells, as it has been previously reported for prostate cancer. First, we analysed the response of breast cancer cells, varying in the initial CD146 mRNA and protein content, to an epigenetic modifier, 5-aza-2-deoxycytidine, and subsequently the methylation status of CD146 gene promoter was investigated, using direct bisulfite sequencing. We observed that treatment with a demethylating agent led to induction of CD146 expression in all analysed breast cancer cell lines, both at the mRNA and protein levels, which was accompanied by an elevated expression of selected mesenchymal markers. Importantly, CD146 gene promoter analysis showed aberrant CpG island methylation in 2 out of 3 studied breast cancer cells lines, indicating epigenetic regulation of the CD146 gene expression. In conclusion, our study revealed for the first time that aberrant methylation may be involved in expression control of CD146, a very potent EMT inducer in breast cancer cells. Altogether, the data obtained may provide basis for novel therapies, as well as diagnostic approaches enabling sensitive and very accurate detection of breast cancer cells.

Key words: CD146 (MCAM, MUC-18), EMT, epigenetic silencing, DNA methylation

Received: 10 November, 2019; revised: 15 November, 2019;

20 November, 2019; accepted: available on-line: 11 December, 2019

छe-mail: kinga.kocemba@uj.edu.pl

*Acknowledgements of Financial Support:

The costs of the article published as a part of the 44th FEBS Congress Kraków 2019 - From molecules to living systems block are financed by the Ministry of Science and Higher Education of the Republic of Poland (Contract 805/P-DUN/2019).

Work presented in this paper was funded by the statutory grants (N41/DBS/000050) and (K/ZDS/008402).

Abbreviations: 5'-Aza-dC, 5-aza-2-deoxycytidine; BS, direct bisulfite sequencing; EMT, Epithelial to Mesenchymal Transition; FBS, fetal bovine serum; HER2, human epidermal growth factor receptor type 2; HPRT1, hypoxanthine Phosphoribosyl transferase 1; MMP2, matrix metalloproteinase-2; MMP9, matrix metalloproteinase-9; VIM, vimentin; TNBC, triple-negative breast cancer; TSS, transcription start sites

\section{INTRODUCTION}

Death of breast cancer patients is mainly caused by metastasis, transforming the locally confined disease into a disseminated and usually incurable one (Felipe Lima et al., 2016). Metastasis can be defined as spreading of cancerous cells into distinct organs, followed by formation of a secondary tumor site. At the molecular level, this process is accompanied by actin cytoskeleton rearrangement and attenuation of cell-cell and cell-extracellular matrix adhesive interactions, which altogether resembles the Epithelial to Mesenchymal Transition (EMT), a morphogenetic process observed during development (Brabletz et al., 2018; Nieto et al., 2016). EMT is driven by mesenchymal transcription factors, including Snail, Slug, Zeb, and Twist, which are responsible for altering the epithelial transcriptional profile into mesenchymal one (Nieto et al., 2016; Felipe Lima et al., 2016). Breast cancer cells undergoing EMT become highly motile and invasive, which is especially apparent in the most aggressive estrogen-negative, progesterone-negative, human epidermal growth factor receptor-negative subtype (ER-/PR-/HER2-), defined as a triple-negative breast cancer (TNBC) (Khaled \& Bidet, 2019; Felipe Lima et al., 2016). TNBC diagnosed patients have a relatively poor prognosis and cannot be subjected to endocrine therapy or therapies directed against human epidermal growth factor receptor type 2 (HER2) (Schneider et al., 2008). Interestingly, recent publication and meta-analysis, covering a high number of solid tumors, revealed a significant association of CD146 protein expression, EMT and poor survival of cancer patients (Zeng et al., 2017). Importantly, independent studies reported that CD146 is highly expressed in TNBC and in metastatic breast cancer, which is contrary to normal tissue and benign tumors (de Kruijff et al., 2018; Garcia et al., 2007; Jang et al., 2015; Zabouo et al., 2009). Moreover, preclinical in vitro and in vivo studies revealed that aberrantly overexpressed CD146 is sufficient to induce acquisition of mesenchymal phenotype in breast cancer cells (Zeng et al., 2012; Imbert et al., 2012). Nevertheless, although overexpressed CD146 is considered an important oncogene in breast carcinogenesis, there is still lack of information about potential regulation of the CD146 gene expression in breast cancer cells. Despite the fact that CD146 is overexpressed in cancer cells, its amplification or mutation has been excluded so far (Wang \& Yan, 2013). In the study presented here, by using breast cancer cell lines as a model, we revealed that CD146 gene promoter is aberrantly methylated in breast cancer cells and the 
Table 1. Sequences of primers used for selected gene expression analyses.

\begin{tabular}{|c|c|c|}
\hline Gene & Length (bp) & Primer Sequences \\
\hline CD146 & 438 & $\begin{array}{l}\text { forward 5'-CCAAGGCAACCTCAGCCATG-3' } \\
\text { reverse 5'-CTCGACTCCACAGTCTGGGA-3' }\end{array}$ \\
\hline HPRT1 & 504 & $\begin{array}{l}\text { forward 5'-TGGCGTCGTGATTAGTGATG-3' } \\
\text { reverse 5'-TATCCAACACTTCGTGGGGT-3' }\end{array}$ \\
\hline SNAI1 & 307 & $\begin{array}{l}\text { forward 5'-GGATCTCCAGGCTCGAAAGG-3' } \\
\text { reverse 5'-CATTCGGGAGAAGGTCCGAG-3' }\end{array}$ \\
\hline TWIST1 & 177 & $\begin{array}{l}\text { forward 5'-CCGTGGACAGTGATTCCCAG-3' } \\
\text { reverse 5'-CCTTTCAGTGGCTGATTGGC-3' }\end{array}$ \\
\hline ZEB1 & 140 & $\begin{array}{l}\text { forward 5'-TCCTCGAGGCACCTGAAGAGG-3' } \\
\text { reverse 5'-CAGAGAGGTAAAGCGTITATAGCC-3' }\end{array}$ \\
\hline SNAI2 & 372 & $\begin{array}{l}\text { forward 5'-GCTACCCAATGGCCTCTCTC-3' } \\
\text { reverse 5'-TTGCCGCAGATCTTGCAAAC-3' }\end{array}$ \\
\hline CADHERIN 1 & 342 & $\begin{array}{l}\text { forward 5'-GCCAAGCAGCAGTACATTCTACACG-3' } \\
\text { reverse } 5^{\prime} \text {-GTCGTTCTTCACGTGCTCAAAATCC-3' }\end{array}$ \\
\hline CADHERIN 2 & 370 & $\begin{array}{l}\text { forward 5'-GCGTTCCTGTTCCACTCATAGGAGG-3' } \\
\text { reverse 5'-GTGCCATTAGCCAAGGGAATTCAGC-3' }\end{array}$ \\
\hline MMP2 & 499 & $\begin{array}{l}\text { forward 5'-TGATGGAGAGGCAGACATCA-3' } \\
\text { reverse 5'-ATACTTCTTGTCGCGGTCGT-3' }\end{array}$ \\
\hline MMP9 & 98 & $\begin{array}{l}\text { forward 5'-CTGAGAACCAATCTCACCGACA-3' } \\
\text { reverse 5'-AGATTTCGACTCTCCACGCA-3' }\end{array}$ \\
\hline
\end{tabular}

others (Statistica, TIBCO Software Inc.). For the purpose of analysis, the non-significant Spearman correlation coefficient was set as zero.

Cell lines and cell culture. MCF7 and T47D breast cancer cell lines, as well as human fibroblast (Hs27) cells, were purchased from the American Type Culture Collection (Rockville, MD, USA). MDAMB-231 breast cancer cell line was purchased from Sigma-Aldrich (St. Louis, MO, USA). All cells were grown in RPMI 1640 medium (Gibco Life Technology), supplemented with 10\% fetal bovine serum (FBS), (EURx, Gdansk, Poland), $2 \mathrm{mmol} / \mathrm{L}$ L-glutamine and 100 units/ $\mathrm{mL}$ penicillin $\mathrm{G}$ and Streptomycin (Sigma-Aldrich, St. Louis, MO, USA). For demethylation study, MCF7, T47D and MDA-MB-231 cells were cultured with and without $10 \mu \mathrm{M}$ 5-aza-2-deoxycytidine (\#PA-03-1241-P, Pol-AURA, Poland) for 6 days, and on day 4 the cell culture media were exchanged for fresh ones, with and without 5-aza-2-deoxycytidine, respectively.

RNA isolation and cDNA synthesis. demethylating agent, 5-aza-2-deoxycytidine, can trigger its expression. According to our knowledge, this is the first study suggesting a role of epigenetics behind the CD146 expression in breast cancer cells. Noteworthy, this finding not only sheds new light on regulation of CD146 expression during breast carcinogenesis, but also provides an important rationale for novel therapeutic strategies in future. If confirmed with primary samples, it may contribute to a significant improvement of the diagnostic process, allowing early and very sensitive detection of breast cancer cells.

\section{MATERIALS AND METHODS}

R2 Database and statistical analysis of publically available data. The $\mathrm{R} 2$ database (http://r2.amc.nl) is a simple to use website-related tool for analysis and data visualization created at the Department of Oncogenomics in the Academic Medical Center (AMC) in Amsterdam, Netherlands. It gives an opportunity to perform different analyses based on well annotated datasets. In case of the study presented here, we selected tool "Correlate Genes" in the panel of 10 breast cancer data sets in order to check correlation between CD146 and the panel of mesenchymal markers. Bonferroni corrected p-value of the Spearman correlation coefficient was used to show that the panel of mesenchymal marker encoding genes significantly correlated with CD146 in breast cancer patients. Since the 9 mesenchymal markers were compared with CD146, p value below 0.0055 was considered as significant $(0.05 / 9)$. The ten breast cancer data sets chosen for analysis included two sets (GSE7396 and GSE46563) composed of only lymph node negative patients and eight data sets (GSE1456, GSE12276, GSE2109, GSE3494, GSE102484, GSE29271, GSE69031, GSE36771) in which lymph node negativity was not the criterion for patient selection. Since the number of genes correlated with CD146 was lower in data sets with confirmed lymph node negativity in comparison to other data sets, we applied cluster analysis (nearest neighbor, Euclidean distance) algorithm to verify if these two data set are indeed different from all the
Total RNA was isolated from harvested cells using Gene Matrix Universal RNA kit (EURx, Gdansk, Poland). NanoDrop ND-1000 Spectrophotometer (NanoDrop Technologies, Wilmington, DE, USA) was used to determine concentration and quality of the isolated RNA. Total RNA $(1 \mu \mathrm{g})$ was used for cDNA synthesis according to the manufacturer's protocol (EURx, Gdansk, Poland).

Reverse transcription polymerase chain reaction (RT-PCR). For PCR reaction, the Color OptiTaq PCR Master Mix (2x) (EURx, Gdansk, Poland) was used according to the manufacturer's protocol. Sequences of reverse and forward primers used in this study are presented in Table 1. For CD146, HPRT1, SNAI1, TWIST, ZEB1 and SNAI2, the PCR reactions were carried out as previously described (Dudzik et al., 2019). For CADHERIN1 and CADHERIN2, the conditions were as following: initial denaturation at $95^{\circ} \mathrm{C}$ for $5 \mathrm{~min}$; followed by 30 cycles: $30 \mathrm{~s}$ at $95^{\circ} \mathrm{C}, 30 \mathrm{~s}$ at $58^{\circ} \mathrm{C}$ and $30 \mathrm{~s}$ at $72^{\circ} \mathrm{C}$; and final extension at $72^{\circ} \mathrm{C}$ for $10 \mathrm{~min}$. For $M M P 2$ and $M M P 9$ the following conditions were applied: initial denaturation at $94^{\circ} \mathrm{C}$ for $3 \mathrm{~min}$; followed by 30 cycles: 45 $\mathrm{s}$ at $94^{\circ} \mathrm{C}, 60 \mathrm{~s}$ at $59^{\circ} \mathrm{C}$ and $60 \mathrm{~s}$ at $72^{\circ} \mathrm{C}$; and final extension at $72^{\circ} \mathrm{C}$ for $5 \mathrm{~min}$. PCR product was visualized on $1.5 \%$ agarose gel stained with ethidium bromide and photographed with Bio-Rad ChemiDoc ${ }^{\mathrm{TM}}$ XRS + System (Bio-Rad, Hercules, CA, USA). HPRT1 was used as internal control to ensure equal sample loading. In case of MDA-MB-231 cells, the RT-PCR analysis of CD146 was performed according to a modified, adjusted PCR protocol in which 25 cycles were used instead of 30 cycles, whereas all other conditions remained unchanged.

The CD146 and HPRT1 primers were manufactured by IBB PAN (Warsaw, Poland). All other primers were purchased from Sigma-Aldrich (St. Louis, MO, USA). The images of the gels were captured using Bio-Rad ChemiDoc $^{\text {TM }}$ XRS + System (Bio-Rad, Hercules, CA, USA) and subsequently analyzed by means of publicly available ImageJ software. All values were normalized to the HPRT1 signal.

Western blot. The cells were lysed and protein concentration was determined as previously described (Kocemba-Pilarczyk et al., 2018). Protein lysates (30 $\mu \mathrm{g}$ ) 
were separated by SDS PAGE (10\%), and subsequently blotted onto nitrocellulose membrane (Thermo Fisher Scientific, MA, USA). After blocking ( $5 \%$ non-fat dry milk), the proteins of interest were detected by the following primary antibodies: anti-CD146 (P1H12, \#13475, Cell Signalling, Danvers, MA, USA, 1:500 in 5\% BSA), anti-N-cadherin (\#610920, BD Pharmigen, 1:1000 in 5\% BSA), anti-E-cadherin (\#610404, BD Pharmigen, 1:1000 in $2 \% \mathrm{BSA}$ ), anti-Vimentin (\#550513, BD Pharmigen, $1: 1000$ in $5 \%$ BSA) and appropriate (anti-mouse or anti-rabbit) HRP-conjugated secondary antibodies (Cell Signalling Technology Inc; 1:2000 in 5\% non-fat milk). Anti-beta-actin primary antibody (Clone AC-15, \#A1978, Sigma-Aldrich, 1:10000 in 5\% non-fat milk) and HRPconjugated secondary antibody (Cell Signalling Technology Inc; $1: 5000$ in 5\% non-fat dry milk) were used to ensure equal protein loading. The signal was visualized by chemiluminescent substrates of different sensitivities, depending on the protein of interest expression level. For low, moderate and high content of analyzed protein, the Signal Fire ${ }^{\mathrm{TM}}$ Elite ELC Reagent (Cell Signaling Technology Inc), LumiGLO Reagent (Cell Signalling Technology Inc) and Amersham ECL ${ }^{\mathrm{TM}}$ Western Blotting Detection Reagents (GE, Healthcare, Chicago, ILL, USA) were used, respectively. Chemiluminescence intensity was recorded with Bio-Rad ChemiDoc ${ }^{\mathrm{TM}} \mathrm{XRS}+$ System (BioRad, Hercules, CA, USA).

Immunofluorescent staining. Cells were washed with Dulbeco's phosphate buffer saline (DPBS) and fixed in 4\% paraformaldehyde for 20 minutes at room temperature. After incubation, the cells were rinsed with DPBS containing $0.1 \%$ Triton X-100 (Sigma-Aldrich) for 5 minutes, and subsequently blocked in 3\% bovine serum albumin (BSA, Sigma-Aldrich) in DPBS without ions for $1 \mathrm{~h}$ at room temperature. Then, the cells were incubated with appropriate primary antibody diluted in $3 \%$ BSA in DPBS for $24 \mathrm{~h}$ at $4^{\circ} \mathrm{C}$. Antibodies were as follows: rabbit anti-E-cadherin antibody (1:200; ab15148; Abcam), mouse anti-vimentin (1:200, V9, sc-6260; Santa Cruze Biotechnology). The cells were washed with DPBS 5 times and then incubated with Hoechst (Sigma-Aldrich), as well as secondary goat anti-rabbit or anti-mouse antibodies conjugated with Alexa Fluor 488 (ThermoFisher) or Alexa Fluor 555 (ThermoFisher) diluted in $3 \%$ BSA in DPBS for $1 \mathrm{~h}$ at room temperature in the dark. The results were analyzed with the IX70 microscope (Olympus Corporation, Tokyo, Japan).

Bisulfite sequencing. Genomic DNA was extracted from MCF7, T47D, MDA-MB-231 and Hs27 cells using GenElute Mammalian Genomic DNA Miniprep Kits (Sigma Aldrich, St. Louis, MO, USA) according to the manufacturer's instructions. Concentration and quality of isolated DNA were assessed by means of NanoDrop ND-1000 Spectrophotometer (NanoDrop Technologies, Wilmington, DE, USA). The DNA was modified, and bisulfite sequencing procedure was carried out as previously described (Dudzik et al., 2019). Primers for bisulfite-converted DNA were as following: forward (5'-GTTGTAAATTGGTTGTAAAGAAGAGTTG-3'), reverse (5'-CAACCT'TCCCAAACCAAAAAC-3'), generating a product of $502 \mathrm{bp}$, which was cut out of the gel, purified according to the manufacturer's instructions (Agarose Out DNA purification kit, EURx, Gdansk, Poland), and subsequently sequenced (Genomed, S.A, Poland). Primers were manufactured by Sigma-Aldrich (St. Louis, MO, USA). The CD146 gene sequence (11q23.3, NCBI/GRCh38.p12, 119305759-119317130) including its promoter was downloaded from the database of $\mathrm{Na}$ tional Centre for Biotechnology Information (NCBI).
The CD146 gene promoter CpG island (cg 21096399, NCBI/GRCh38.p12, 119317381-119316788) encompasses exon 1, including transcriptional (NCBI/GRCh38. p12, 119317130) and translational (NCBI/GRCh38.p12, 119317101) start sites, as well as the fragment of 5' upstream coding sequence (Kocemba et al., 2016), which altogether is characteristic for genes that are transcriptionally silenced by an epigenetic mechanism (Feltus et al., 2003). The area of $\mathrm{CpG}$ island selected for methylation analysis ( +238 to -162 in relation to the transcription start sites, TSS), containing representative $50 \mathrm{CpG}$ sites (out of the $62 \mathrm{CpG}$ dinucleotides present in the specific $\mathrm{CpG}$ island), covered exon 1 with transcriptional and translational start sites.

\section{RESULTS}

\section{The relation between CD146 and mesenchymal profile in breast cancer cell lines}

In spite of a number of studies confirming the relationship between CD146 and tumor invasiveness, poor prognosis or mesenchymal features of breast cancer cells, a recent work by de Kruijff et al. questioned the existence of EMT-CD146 association in a group of analysed breast cancer patients (de Kruijff et al., 2018). Although these authors indeed reported CD146 as a prognostic factor for metastasis free and overall survival in a univariable analysis, they did not find a correlation between CD146 and mesenchymal markers at the level of mRNA expression (de Kruijff et al., 2018). In order to perform an independent verification, we analysed the relation between CD146 expression and the panel of mesenchymal markers in 10 independent transcriptomic data sets of breast cancer patients. The patients' data sets selected for this analysis included 2 data sets containing breast cancer patients with confirmed lymph node negativity (lymph node negative data sets) and 8 data sets in which patients with lymph node negative and positive status were combined (non-lymph node negative data sets). As shown in Table 2, at least 6 out of 9 mesenchymal markers were significantly correlated with CD146 in non-lymph node negative data sets, whereas in lymph node negative datasets we have found only 2 mesenchymal markers correlated with CD146 in the GSE7396 data set, and only one (vimentin) correlated with CD146 in the GSE46563 data set. In fact, vimentin was the only gene correlated

Table 2. Correlation analysis between CD146 and nine selected mesenchymal markers in 10 independent transcriptomic data sets of breast cancer patients.

White rectangle indicates lack of significance, grey rectangle indicates a significant Spearman correlation coefficient below 0.5 , black rectangle indicates a significant Spearman correlation coefficient above 0.5. Bonferroni corrected $p$-value below 0.0055 was considered as statistically significant.

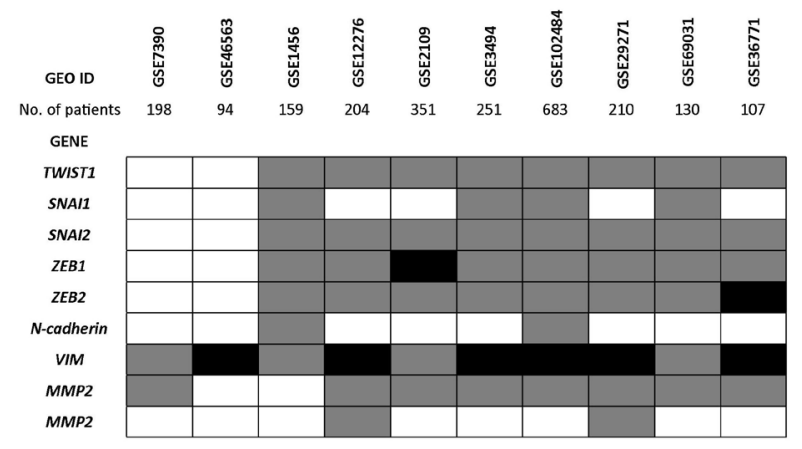


A
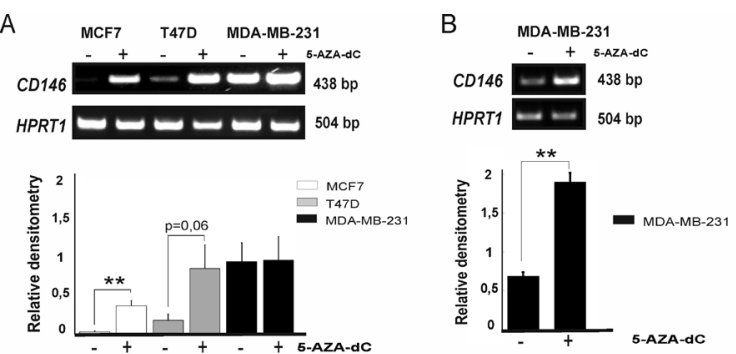

C

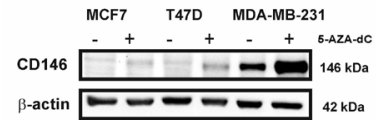

Figure 1. The effect of demethylating agent, 5-aza-2-deoxycytidine, on expression of CD146 in breast cancer cell lines at the mRNA and protein level.

(A) upper panel: Representative results of RT-PCR analysis for CD146 expression in breast cancer cell lines cultured in the absence and presence of $10 \mu \mathrm{M}$ 5-aza-2-deoxycytidine for $144 \mathrm{~h}$. Lower panel: Graph presenting densitometry analysis of three independent experiments. Mean values \pm S.E.M. (standard error of the mean) after normalization to HPRT signal are shown. ${ }^{* *} p<0.01$, by student's $t$-test, $p$-value between 0.05 and 0.1 was considered as an indication of a trend. (B) upper panel: Representative results of RT-PCR analysis for CD146 expression in MDA-MB-231 breast cancer cell line cultured in the absence and presence of $10 \mu \mathrm{M}$ 5-aza-2-deoxycytidine for $144 \mathrm{~h}$. Lower panel: Graph presenting densitometry analysis of three independent experiments. Mean values \pm S.E.M. after normalization to HPRT signal are shown. ${ }^{* *} p<0.01$, by student's $t$-test. (C) Western blot analysis of CD146 protein expression level in breast cancer cell lines cultured in the absence and presence of $10 \mu \mathrm{M} 5$-aza-2-deoxycytidine for $144 \mathrm{~h}$. Representative experiment of three independent ones is shown.

with CD146 in all analysed data sets, regardless of the lymph node status. In order to verify if two lymph node negative data sets are different from the others, cluster analysis was performed. This approach further confirmed that two lymph node negative data sets clustered together as being different from all the others. Thus, the results of our analysis clearly demonstrated, based on transcriptomic data, the existence of association between CD146 expression, mesenchymal profile and the progression of breast cancer.

\section{The effect of 5-aza-2-deoxycytidine on expression of CD146 in breast cancer cell lines}

In order to check whether CD146 expression is regulated at the transcriptional level by an epigenetic modifier, 5-aza-2-deoxycytidine, as it has been shown previously in prostate cancer cell lines (Dudzik et al., 2019), two epithelial breast cancer cell lines (MCF7 and T47D) (Dai et al., 2017) and a mesenchymal one (MDA-MB-231) (Dai et al., 2017) were cultured with and without 5-aza-2-deoxycytidine $(10 \mu \mathrm{M})$ for 6 days, as previously described, followed by CD146 expression analysis at the mRNA and protein level. As expected, basal expression of CD146 was low in the MCF7 and T47D cancer cell lines with epithelial characteristics, and high in the MDA-MB-231 cell line with mesenchymal expression profile. As shown in Fig. 1, CD146 expression was apparently induced in the MCF7 and T47D cells, whereas in MDA-MB-231 its high basal expression disabled correct assessment of expression difference between the control and 5-aza-2-deoxycytidine treated cells. Thus, in order to verify if high CD146 expression in MDA-MB-231 is still further inducible, we performed an expression analysis according to an adjusted, modified
PCR protocol, as described in the Materials and Methods section. Interestingly, in MD-MB-231, the CD146 expression was also significantly induced, regardless of the high basal level and the mesenchymal characteristics of these cells (Fig. 1B). As for the protein analysis (Fig. 1C), the demethylating compound had significantly induced CD146 expression in all analysed cell lines. This effect was clearly visible in the epithelial lines with very low basal expression of CD146, as well as in the MDAMB-231 cells, where the relatively high basal expression was still apparently inducible, in accordance with the data obtained at the mRNA level. Altogether, this data suggest that epigenetic silencing plays an important role in the control of CD146 expression in breast cancer cells with epithelial characteristics (MCF7 and T47D), whereas in mesenchymal breast cancer cell line (MDA-MB-231) expression of CD146 is apparently not fully unleashed and still partially trapped by the epigenetic mechanism.

The effect of 5-aza-2-deoxycytidine on induction of the mesenchymal profile

To determine if CD146 gene expression increase is associated with a mesenchymal profile induction, we analysed the influence of an epigenetic modifier, 5 aza2-deoxycytidine $(10 \mu \mathrm{M})$, on expression panel of mesenchymal markers: Slug (SNAI2), Twist1 (TWIST1), Zeb1 (ZEB1), Snail (SNAI1), N-cadherin (CADHERIN 2), Vimentin (VIM), matrix metalloproteinase-2 (MMP2) and matrix metalloproteinase-9 (MMP9), and an epithelial marker E-cadherin (CADHERIN 1). The mesenchymal markers subjected to this analysis were selected based on the expression correlation studies performed in breast cancer patients (Table 2). As shown in Fig. 2A, treatment of breast cancer cell lines with the demethylating agent resulted in gene expression changes at the mRNA level for several mesenchymal markers, such as SANI1,

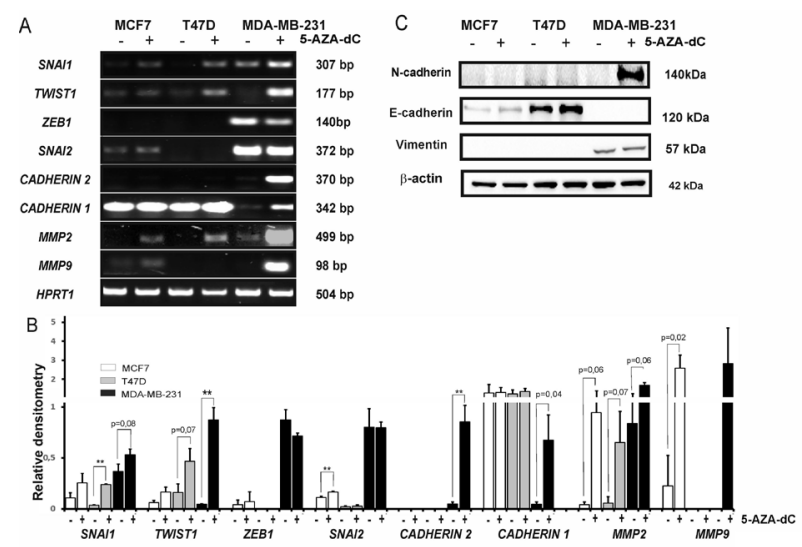

Figure 2. The effect of demethylating agent, 5-aza-2-deoxycytidine, on expression of selected mesenchymal markers and $E$ cadherin, in breast cancer cell lines at the mRNA and protein level.

(A) Representative results of RT-PCR analysis of mesenchymal markers and E-cadherin expression in breast cancer cell lines cultured in the absence and presence of $10 \mu \mathrm{M}$ 5-aza-2-deoxycytidine for 144 h. (B) Densitometric quantification of mRNA expression of selected EMT-related genes in breast cancer cell lines cultured in the absence and presence of $10 \mu \mathrm{M}$ 5-aza-2-deoxycytidine for 144 h. Data are shown as mean values \pm S.E.M. of 3 independent experiments after normalization to respective HPRT1 levels. ${ }^{* *} p<0.01$, by student's t-test, $p$-value between 0.05 and 0.1 was considered as an indication of a trend. (C) Western blot analysis of $\mathrm{N}$-cadherin, $\mathrm{E}$-cadherin and Vimentin protein expression in breast cancer cell lines cultured in the absence and presence of $10 \mu \mathrm{M}$ 5-aza-2-deoxycytidine for $144 \mathrm{~h}$. Representative experiment of two independent ones is shown. 


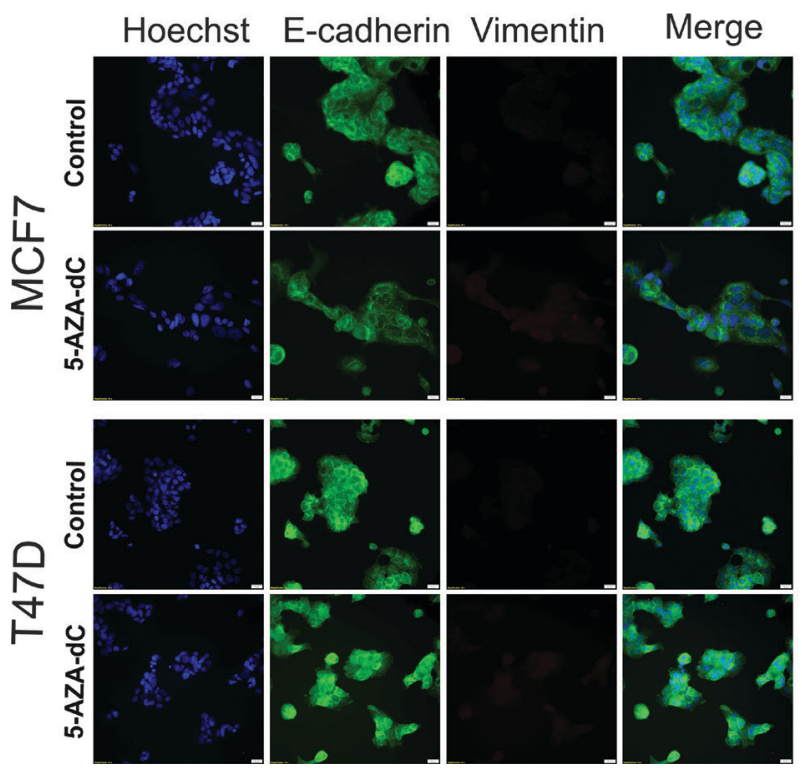

Figure 3. The effect of demethylating agent, 5-aza-2-deoxycytidine, on cell morphology and expression of E-cadherin and Vimentin in epithelial breast cancer cell lines (MCF7 and T47D), assessed by immunofluorescence.

Immunofluorescence staining of E-cadherin and vimentin in breast cancer cell lines (MCF7 an dT47D) cultured in the absence and presence of $10 \mu \mathrm{M}$ 5-aza-2-deoxycytidine for $144 \mathrm{~h}$.
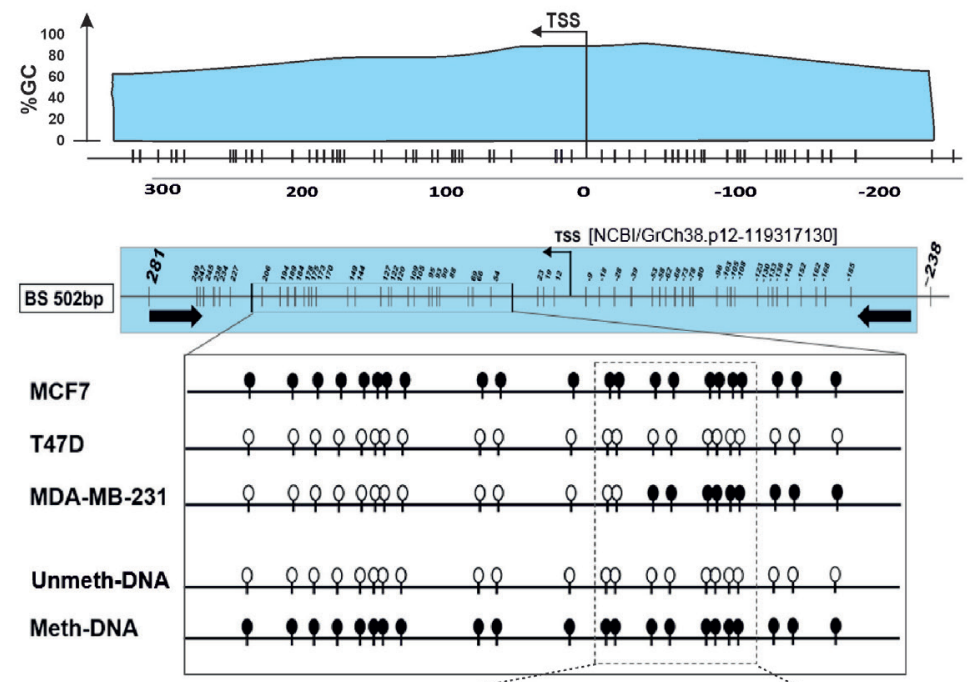

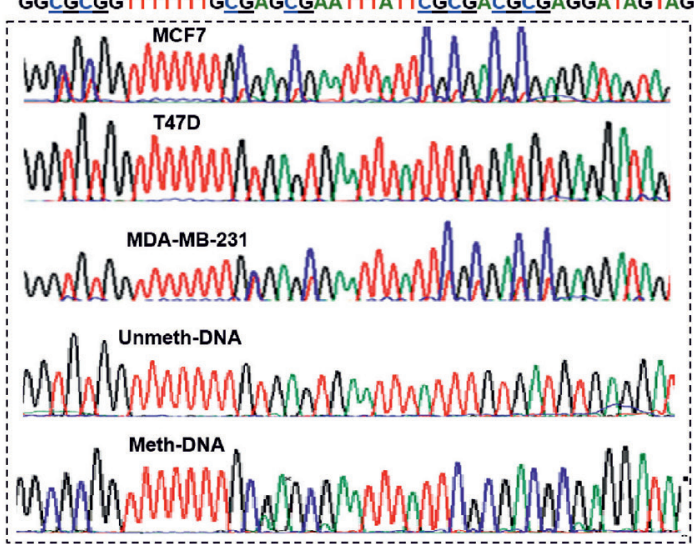

SNAI2, TWIST1, MMP2, MMP9 and CADHERIN 2, whereas densitometry analysis of three independent experiments (Fig. 2B) confirmed significant changes/or indication of the trend for SNAI1 (T47D, MDA-MB-231), TWIST1 (T47D, MDA-MB-231), SNAI2 (MCF7), CADHERIN 2 (MDA-MB-231), MMP2 (MCF7, T47D, MDA-MB-231) and MMP9 (MCF7). As for the epithelial marker, CADHERIN 1, the basal mRNA expression was high in cell lines with epithelial characteristics and low in MDA-MB-231. Of note, in all three independent experiments, we observed an increase of CADHERIN 1 expression at the mRNA level in the MDA-MB-231 cells, regardless of the induction of mesenchymal marker expression (SNAI1 and TWIST1) in these cells, which are the well known transcription factors inhibiting the CADHERIN 1 gene promoter. Concerning protein analysis, E-cadherin was not visibly induced in MDAMB-231, opposite to N-cadherin, whose expression was reproducibly enhanced after treatment with 5-aza-2-deoxycytidine, in two independent experiments. As for vimentin, it was apparently expressed only in the MDAMB-231 cell line and not further induced by treatment with 5-aza-2-deoxycytidine. Undoubtedly, however, the fact that an epigenetic mechanism appears to control expression of such an important oncogene in breast cancer seems to be of high importance. To further determine if 5-aza-2-deoxycytidine, resulting in CD146 induction, may as well trigger the changes in morphology of epithelial breast cancer cells and/or induce the protein expression changes at the level of a single cell, we performed an immunofluorescence analysis of E-cadherin and Vimentin in the MCF7 and T47D cells cultured for 6 days with and without 5-aza-2-deoxycytidine. As determined in Fig. 3, we neither observed an apparent changes in cell morphology, nor, in accordance with the Western blot results, an alteration in the E-cadherin and Vimentin cell content.

The analysis of CD146 gene promoter methylation in breast cancer cell lines

Since presence of the $\mathrm{CpG}$ island in CD146 gene promoter has been already described and its proper location reported by our group (Kocemba et al., 2016), we subjected the CD146 promoter region, including the $\mathrm{CpG}$ island encompassing the transcriptional start site in exon 1, to methylation analysis using direct bisulfitesequencing (BS) of PCR products. DNA isolated from human fibroblasts and in vitro

Figure 4. Pattern of $\mathrm{CpG}$ island methylation in selected region of the CD146 gene promoter. Schematic representation of the CpG island area analyzed in the CD146 gene promoter. Arrows mark the positions of bisulfite sequencing primers. Each of the CpG dinucleotides is presented as a vertical line. The figure shows a representative fragment containing representative $22 \mathrm{CpG}$ sites out of 50 analyzed ones. Electropherograms of bisulfite modified DNA from a representative CD146 gene promoter CpG island fragment in MCF7, T47D, MDA-MB-231 is shown together with DNA isolated from human fibroblasts and in vitro methylated DNA used as an unmethylated and methylated control DNA, respectively. White circles indicate unmethylated CpG sites, black circles indicate the presence of methylation in $\mathrm{CpG}$ sites detected by direct Sanger sequencing of the bisulfite-modified DNA. 
methylated DNA was modified and subsequently used in BS sequencing as unmethylated and methylated control, respectively, to validate our experimental set-up. BS analysis revealed that two (MCF7 and MDA-MB-231) out of the three breast (Fig. 4) cancer cell lines tested (MCF7, T47D and MDA-MB-231) displayed hypermethylation of CpG island in the CD146 promoter area, whereas T47D was methylation-free. Notably, BS analysis revealed heterogeneous methylation in MCF7 and MDA-MB-231, suggesting clonal variation in the methylation pattern of the $\mathrm{CpG}$ island area in these cell lines. Importantly, aberrant methylation detected in the $\mathrm{CpG}$ island of the CD146 gene (Fig. 4) was present in the promoter area encompassing exon one, the region reported previously as crucial for epigenetic silencing of gene expression (Brenet et al., 2011).

\section{DISCUSSION}

To our knowledge, this is the first study showing methylation of the CD146 promoter region in breast cancer cells, and concomitantly suggesting that an epigenetic mechanism may be important in expression control of this relevant metastasis related-oncogene. In our previous paper, using prostate cancer cell lines, we have shown that the CD146 gene is induced by a demethylating compound, 5 aza-2-deoxycytidine, however, analysis of the CpG island region in those cells did not confirm presence of promoter methylation (Dudzik et al., 2019). Of note, the link between epigenetics and expression of the CD146 gene was reported previously in prostate cancer patients but the authors of that study incorrectly localized the $\mathrm{CpG}$ island in the CD146 gene promoter (Liu et al., 2008), and in consequence misinterpreted the results suggesting, contrary to the dogma, that presence of the $\mathrm{CPG}$ island methylation correlates with increase in the CD146 gene expression in prostate cancer cells (Liu et al., 2008). This mistake was pointed out in a Letter to the Editor published in The Prostate in 2016 (Kocemba et al., 2016), also determining proper localization of the $\mathrm{CpG}$ island in the CD146 gene promoter (Kocemba et al., 2016). So far, in literature, the relation between DNA methylation in the promoter area (regardless of presence of a CpG island) and gene expression inactivation has been well confirmed (Han et al., 2011; Smith \& Meissner, 2013). It has been verified that canonical promoter methylation is associated with expression of imprinted genes, the process of $\mathrm{X}$ chromosome inactivation and tissue specific gene regulation (Smith \& Meissner, 2013; Urbano et al., 2019). On the other hand, CpG islands observed around transcription start sites in $50 \%$ of gene promoters are methylation free in non-cancerous cells, regardless of the expression state of the gene of interest (Jones, 2012). However, de novo hypermethylation of canonical CpG islands has been observed in cancer cells in association with inactivation of gene expression (Jones \& Baylin, 2007). Although we observed the CpG island methylation of the CD146 promoter in 2 out of 3 analysed breast cancer cell lines, it does not have to be the only and unique methylated region which controls CD146 expression, since in the T47D breast cancer cell line and three prostate cancer cell lines published previously (Dudzik et al., 2019), the CD146 expression was induced by demethylating treatment, while the analysed $\mathrm{CpG}$ island was methylation free. Importantly, recent studies revealed that methylation in the upstream and downstream CpG island shores may inhibit gene transcription in cancer cells, whereas the $\mathrm{CpG}$ island itself can remain methylation free (Rao et al., 2013; Irizarry et al., 2009). Even more, in a recent publication, Skvortsova et al. revealed that methylation present in the CpG island shores, particularly in the breast cancer cells, is involved in transcriptional silencing of gene expression. These data clearly confirm that promoter methylation in silenced genes in cancer does not have to be confined only to the CpG island (Skvortsova et al., 2019). To verify exactly which promoter part must be demethylated to unleash the CD146 expression would be definitely of high importance. Whereas overexpressed CD146 is considered to be oncogenic in breast cancer cells, as reported by independent studies (Zeng et al., 2012; de Kruijff et al., 2018; Garcia et al., 2007; Zabouo et al., 2009; Imbert et al., 2012), the methylation-based silencing of its expression in these cells seems to be quite surprising. According to our hypothesis, at the beginning of the disease CD146 may be targeted for aberrant promoter methylation/or it has been already methylated in consequence of tissue specific epigenetic silencing, whereas the loss of methylation in advanced tumour unleashes CD146 expression, leading to metastasis. The fact that CD146-dependent induction of a mesenchymal profile has been already described in the literature (Imbert et al., 2012; Zeng et al., 2012; Zabouo et al., 2009) allows us to speculate that increase in expression of mesenchymal related genes may result from CD146 induction, triggered by an epigenetic modifier. On the other hand, we cannot exclude that the epigenetic modifier, in parallel to the increase in CD146 expression, directly induces mesenchymal profile in the breast cancer cell lines, as the EMT induction in consequence of 5-aza-2-deoxycytidine application has been already reported for breast cancer cells (Su et al., 2018). Definitely, more research is needed to elucidate the independent role of CD146, expression triggered by an epigenetic modifier, in EMT induction in breast cancer cells. Interestingly, in a mesenchymal cell line (MDA-MB-231), we observed an increase in the epithelial marker, E-cadherin, at the mRNA level, which can be the direct consequence of demethylating agents, since epigenetic silencing of E-cadherin expression by aberrant promoter methylation has been reported previously in cancer cells of mesenchymal characteristics and what is more, exactly in MDA-MB-231. Thus, it can be concluded that our results for CADHERIN 1 in MDAMB-231 simply indicate a proper experimental setup for the demethylating treatment. It is also important to mention that Imbert et al., after overexpression of CD146 in the MCF7 cell line, reported lack of alteration in TWIST1, SNAI1, MMP2 and MMP9 expression and an increase in CADHERIN 2 and VIM (Imbert et al., 2012), whereas changes in the mesenchymal markers' profile were completely different in our current study, which is most probably the consequence of combined action of CD146 and the demethylating agent. Undoubtedly, however, showing that expression of CD146 is controlled by an epigenetic mechanism seems to be the most important message from our study. Moreover, our analysis also suggests coexistence of cells with methylated and unmethylated CD146 gene promoter, which indicates that alterations in the CD146 gene promoter methylation may reflect the process of clonal selection during breast cancer progression. In this context, the aspect of epigenetic control of CD146 expression takes on a particular significance in research centred on application of 5-aza2-deoxycytidine in treatment of breast cancer patients, and should be considered in planning therapy combined with epigenetic modifiers. It is especially important, since this kind of therapy for breast cancer patients has been 
currently investigated (Connolly et al., 2017). Our data undoubtedly requires further verification to potentially reveal the scenario of methylation changes in the CD146 gene promoter during development of breast cancer progression, as we cannot exclude that CD146 acts as a tumour suppressor at the initial stage of carcinogenesis, as suggested by the study of Shih and others (Shih et al., 1997), and turn into an oncogene in the advance stage, when the transcriptional profile of cancer cells is significantly altered. Overall, our study provides strong basis for further research on epigenetic regulation of CD146, which can significantly contribute to novel therapies and/or development of DNA methylation-based assay for sensitive detection of breast cancer cells.

\section{REFERENCES}

Brabletz T, Kalluri R, Nieto MA, Weinberg RA (2018) EMT in cancer. Nat Rev Cancer 18: 128-134. https://doi.org/10.1038/nrc.2017.118

Brenet F, Moh M, Funk P, Feierstein E, Viale AJ, Socci ND, Scandura JM (2011) DNA methylation of the first exon is tightly linked to transcriptional silencing. PLoS One 6: e14524. https://doi. org/10.1371/journal.pone.0014524

Connolly RM, Li H, Jankowitz RC, Zhang Z, Rudek MA, Jeter SC, Slater SA, Powers P, Wolff AC, Fetting JH, Brufsky A, Piekarz R, Ahuja N, Laird PW, Shen H, Weisenberger DJ, Cope L, Herman JG, Somlo G, Garcia AA, Jones PA, Baylin SB, Davidson NE, Zahnow CA, Stearns V (2017) Combination epigenetic therapy in advanced breast cancer with 5-azacitidine and entinostat: A phase II national cancer institute/stand up to cancer study. Clin Cancer Res 23: 2691-2701. https://doi.org/10.1158/1078-0432.ccr-16-1729

Dai X, Cheng H, Bai Z, Li J (2017) Breast cancer cell line classification and its relevance with breast tumor subtyping. J Cancer 8: 3131-3141. https://doi.org/10.7150/jca.18457

de Kruijff IE, Timmermans AM, den Bakker MA, Trapman-Jansen A, Foekens R, Meijer-Van Gelder ME, Oomen-de Hoop E, Smid M, Hollestelle A, van Deurzen CHM, Foekens JA, Martens JWM, Sleijer S (2018) The prevalence of CD146 expression in breast cancer subtypes and its relation to outcome. Cancers (Basel) 10. https://doi, org/10.3390/cancers10050134

Dudzik P, Trojan SE, Ostrowska B, Zemanek G, Dulinska-Litewka J, Laidler P, Kocemba-Pilarczyk KA (2019) The epigenetic modifier 5-aza-2-deoxycytidine triggers the expression of CD146 gene in prostate cancer cells. Anticancer Res 39: 2395-2403. https://doi. org/10.21873/anticanres.13357

Felipe Lima J, Nofech-Mozes S, Bayani J, Bartlett JM (2016) EMT in breast carcinoma - A review. J Clin Med 5. https://doi.org/10.3390/ jcm5070065

Feltus FA, Lee EK, Costello JF, Plass C, Vertino PM (2003) Predicting aberrant $\mathrm{CpG}$ island methylation. Proc Natl Acad Sci U S 100: 12253-12258. https://doi.org/10.1073/pnas.2037852100

Garcia S, Dales JP, Charafe-Jauffret E, Carpentier-Meunier S, Andrac-Meyer L, Jacquemier J, Andonian C, Lavaut MN, Allasia C, Bonnier P, Charpin C (2007) Poor prognosis in breast carcinomas correlates with increased expression of targetable CD146 and c-Met and with proteomic basal-like phenotype. Hum Pathol 38: 830-841. https://doi.org/10.1016/j.humpath.2006.11.015

Han H, Cortez CC, Yang X, Nichols PW, Jones PA, Liang G (2011) DNA methylation directly silences genes with non-CpG island promoters and establishes a nucleosome occupied promoter. Hum Mol Genet 20: 4299-4310. https://doi.org/10.1093/hmg/ddr356

Imbert AM, Garulli C, Choquet E, Koubi M, Aurrand-Lions M, Chabannon C (2012) CD146 expression in human breast cancer cell lines induces phenotypic and functional changes observed in Epithelial to Mesenchymal Transition. PLoS One 7: e43752. https://doi. org/10.1371/journal.pone.0043752

Irizarry RA, Ladd-Acosta C, Wen B, Wu Z, Montano C, Onyango P, Cui H, Gabo K, Rongione M, Webster M, Ji H, Potash J, Sabunciyan S, Feinberg AP (2009) The human colon cancer methylome shows similar hypo- and hypermethylation at conserved tissue-specific CpG island shores. Nat Genet 41: 178-186. https://doi. org/10.1038/ng.298
Jang MH, Kim HJ, Kim EJ, Chung YR, Park SY (2015) Expression of epithelial-mesenchymal transition-related markers in triple-negative breast cancer: ZEB1 as a potential biomarker for poor clinical outcome. Hum Pathol 46: 1267-1274. https://doi.org/10.1016/j. humpath.2015.05.010

Jones PA (2012) Functions of DNA methylation: islands, start sites, gene bodies and beyond. Nat Rev Genet 13: 484-492. https://doi. org $/ 10.1038 / \operatorname{nrg} 3230$

Jones PA, Baylin SB (2007) The epigenomics of cancer. Cell 128: 683692. https://doi.org/10.1016/j.cell.2007.01.029

Khaled N, Bidet Y (2019) New insights into the implication of epigenetic alterations in the EMT of triple negative breast cancer. Cancers (Basel) 11. https://doi.org/10.3390/ cancers11040559

Kocemba KA, Dudzik P, Ostrowska B, Laidler P (2016) Incorrect analysis of MCAM gene promoter methylation in prostate cancer. Prostate 76: 1464-1465. https://doi.org/10.1002/pros.23210

Kocemba-Pilarczyk KA, Ostrowska B, Trojan S, Aslan E, Kusior D, Lasota M, Lenouvel C, Dulinska-Litewka J (2018) Targeting the hypoxia pathway in malignant plasma cells by using 17-allylamino17-demethoxygeldanamycin. Acta Biochim Pol 65: 101-109. https:// doi.org/10.18388/abp.2017_1630

Liu JW, Nagpal JK, Jeronimo C, Lee JE, Henrique R, Kim MS, Ostrow KL, Yamashita K, van Criekinge V, Wu G, Moon CS, Trink B, Sidransky D (2008) Hypermethylation of MCAM gene is associated with advanced tumor stage in prostate cancer. Prostate 68: 418426. https://doi.org/10.1002/pros.20709

Nieto MA, Huang RY, Jackson RA, Thiery JP (2016) EMT: 2016. Cell 166: 21-45. https://doi.org/10.1016/i.cell.2016.06.028

Rao X, Evans J, Chae H, Pilrose J, Kim S, Yan P, Huang RL, Lai HC, Lin H, Liu Y, Miller D, Rhee JK, Huang YW, Gu F, Gray JW, Huang TM, Nephew KP (2013) CpG island shore methylation regulates caveolin-1 expression in breast cancer. Oncogene 32: 45194528. https://doi.org/10.1038/onc.2012.474

Schneider BP, Winer EP, Foulkes WD, Garber J, Perou CM, Richardson A, Sledge GW, Carey LA (2008) Triple-negative breast cancer: risk factors to potential targets. Clin Cancer Res 14: 8010-8018. https://doi.org/10.1158/1078-0432.ccr-08-1208

Shih LM, Hsu MY, Palazzo JP, Herlyn M (1997) The cell-cell adhesion receptor Mel-CAM acts as a tumor suppressor in breast carcinoma. Am J Pathol 151: 745-751

Skvortsova K, Masle-Farquhar E, Luu PL, Song JZ, Qu W, Zotenko E, Gould CM, Du Q, Peters TJ, Colino-Sanguino Y, Pidsley R, Nair SS, Khoury A, Smith GC, Miosge LA, Reed JH, Kench JG, Rubin MA, Horvath L, Bogdanovic O, Lim SM, Polo JM, Goodnow CC, Stirzaker C, Clark SJ (2019) DNA hypermethylation encroachment at $\mathrm{CpG}$ island borders in cancer is predisposed by $\mathrm{H} 3 \mathrm{~K} 4$ monomethylation patterns. Cancer Cell 35: 297-314. e298. https://doi. org/10.1016/j.ccell.2019.01.004

Smith ZD, Meissner A (2013) DNA methylation: roles in mammalian development. Nat Rev Genet 14: 204-220. https://doi.org/10.1038/ nrg3354

Su Y, Hopfinger NR, Nguyen TD, Pogash TJ, Santucci-Pereira J, Russo J (2018) Epigenetic reprogramming of epithelial mesenchymal transition in triple negative breast cancer cells with DNA methyltransferase and histone deacetylase inhibitors. I Exp Clin Cancer Res 37: 314. https://doi.org/10.1186/s13046-018-0988-8

Urbano A, Smith J, Weeks RJ, Chatterjee A (2019) Gene-specific targeting of DNA methylation in the mammalian genome. Cancers (Basel) 11. https://doi.org/10.3390/cancers11101515

Wang Z, Yan X (2013) CD146, a multi-functional molecule beyond adhesion. Cancer Lett 330: 150-162. https://doi.org/10.1016/j.canlet.2012.11.049

Zabouo G, Imbert AM, Jacquemier J, Finetti P, Moreau T, Esterni B, Birnbaum D, Bertucci F, Chabannon C (2009) CD146 expression is associated with a poor prognosis in human breast tumors and with enhanced motility in breast cancer cell lines. Breast Cancer Res 11: R1. https://doi.org/10.1186/bcr2215

Zeng P, Li H, Lu PH, Zhou LN, Tang M, Liu CY, Chen MB (2017) Prognostic value of CD146 in solid tumor: A systematic review and meta-analysis. Sci Rep 7: 4223. https://doi.org/10.1038/s41598-01701061-3

Zeng Q, Li W, Lu D, Wu Z, Duan H, Luo Y, Feng J, Yang D, Fu L, Yan X (2012) CD146, an epithelial-mesenchymal transition inducer, is associated with triple-negative breast cancer. Proc Natl Acad Sci US A 109: 1127-1132. https://doi.org/10.1073/pnas.1111053108 